\section{Sunitinib adjuvant bei lokal fortgeschrittenem RCC}

\author{
Nachdem sich die antiangiogene Therapie, z. B. mit Hemmung des VEGFR- \\ Weges durch Sorafenib oder Sunitinib, in der Behandlung des metastasierten \\ RCC als effektiv erwiesen hat, sollte in der S-TRAC-Studie der Stellenwert von \\ Sunitinib adjuvant nach Nephrektomie bestimmt werden.
}

W ährend frühe Stadien eines Nierenzellkarzinoms (RCC) eine günstige Prognose haben, sinkt die 5-JahresÜberlebensrate bei lokal fortgeschrittenen RCC (Stadium III) auf $53 \%$. Nach der Operation stellt sich daher die Frage nach einer adjuvanten Therapie. Deshalb wurden von 2007-2011 615 Patienten mit klarzelligem Hochrisiko-RCC im Stadium III (T3 und/oder N1) in die Phase-IIIStudie S-TRAC aufgenommen. Die Patienten erhielten für maximal $1 \mathrm{Jahr}$ entweder Sunitinib (SUN; 4 Wochen, danach 2 Wochen Pause) oder Placebo. Primärer Endpunkt war das krankheitsfreie Überleben (DFS), sekundäre Studienziele das vom betreuenden Arzt bestimmte DFS, das Gesamtüberleben (OS) und die Sicherheit.
Das DFS war unter SUN im Vergleich zu Placebo signifikant länger (6,8 vs. 5,6 Jahre; Hazard Ratio 0,76; p = 0,03). Bestimmten die betreuenden Ärzte das DFS selbst, ergab sich ebenfalls ein numerischer Unterschied zugunsten von SUN (6,5 vs. 4,5 Jahre; nicht signifikant). Das OS kann derzeit noch nicht ausgewertet werden.

Dosisreduktionen wegen Nebenwirkungen waren unter SUN mit 34,3\% viel häufiger als unter Placebo mit $2 \%$. Dasselbe gilt für Therapieunterbrechungen $(46,4$ vs. $13,2 \%)$ und -abbrüche $(28,1$ vs. $5,6 \%)$. Grad-3/4-Nebenwirkungen traten mit $60,5 \%$ unter SUN wesentlich häufiger auf als unter Placebo mit 19,4\%. Bei den Nebenwirkungen von SUN stand die Diarrhö (56,9\% der Patienten) im Vordergrund; Grad 3 wurde jedoch nur bei 3,9\% registriert. Sehr häufig waren außerdem ein Hand-Fuß-Syndrom mit 50,3\% und Hypertonie mit 36,9\%; auch hier waren die Schweregrade $\geq 3$ mit 16,0 bzw. 7,8\% deutlich seltener.

Bei der Lebensqualität wurden ebenfalls signifikante Unterschiede zu Ungunsten der SUN-Therapie festgestellt. Diese waren aber numerisch relativ gering und erlangten lediglich bei der Diarrhö klinische Relevanz.

Fazit: Nach Nephrektomie wegen eines klarzelligen RCC im Stadium III verlängerte die adjuvante Sunitinib-Therapie in einer großen Phase-III-Studie signifikant das DFS. Diesem positiven Ergebnis steht aber eine relevante Zunahme an Nebenwirkungen gegenüber. Aussagen bezüglich etwaiger Effekte auf das OS sind noch nicht möglich.

Brigitte Schalhorn

Ravaud A et al. Adjuvant sunitinib in high-risk renal-cell carcinoma after nephrectomy. $\mathrm{N}$ Engl J Med. 2016;375(23):2246-54.

\section{Neutrophilen-Lymphozyten-Quotient bei Harnblasentumoren - nicht prädiktiv}

\begin{abstract}
In der Therapie des Harnblasenkarzinoms (BC) wäre ein Biomarker zur Einschätzung des Risikos hochwillkommen. In einer Reihe von Untersuchungen erschien der Neutrophilen-Lymphozyten-Quotient (NLR) als vielversprechender Kandidat. Diese Annahme konnte jedoch nicht bestätigt werden.
\end{abstract}

D ie präoperative Risikostratifizierung ist beim BC eine Herausforderung. In früheren Studien schien der NLR hier prognostischen Wert zu haben, aber viele der Untersuchungen hatten methodische Mängel. Daher wurde nun in einer ausgefeilteren Analyse untersucht, ob der NLR ein Prognosemarker für das Gesamtüberleben (OS) nach kurativer Behandlung oder ein prädiktiver Marker für das Überleben nach neoadjuvanter Chemotherapie (NAC) sein könnte.

Die ungeplante sekundäre Analyse stützt sich auf die prospektiv erhobenen Daten der Phase-III-Studie SWOG 8710, in der von 1987-1998 bei 317 Patienten mit muskelinvasivem BC (T2-T4aN0) das Gesamtüberleben (OS) nach Zystektomie mit oder ohne NAC untersucht worden war. Für die Testung des prognostischen Werts des NLR wurden Daten von 230 Patienten ausgewertet, für den prädiktiven Wert Daten von $263 \mathrm{~Pa}$ tienten.

Nach median 18,6 Jahren wurden 172 Todesfälle in der prognostischen und 205 in der prädiktiven Kohorte registriert. In der multivariaten Analyse war der NLR nicht prognostisch für das OS (Hazard Ratio [HR] 1,04; p = 0,24). Darüber hinaus zeigte sich auch kein prädiktiver Wert bezüglich des Nutzens der NAC (HR 1,01; $p=0,86)$. Von vorab definierten potenziellen Einflussfaktoren waren ein höheres Lebensalter (HR 1,05; $\mathrm{p}<0,001)$ und die alleinige Operation ohne NAC (HR 1,39; $p=0,03$ ) mit einem schlechteren OS assoziiert.

Die Abweichungen gegenüber früheren Studienergebnissen könnten verschiedene Ursachen haben: Alle Analysen beruhten auf Beobachtungsstudien, die per se keine ausreichende Evidenz erreichen. Eine andere Ursache könnte der Reporting Bias sein. Die größte Rolle spielt aber wahrscheinlich der Publication Bias, was für Biomarkerstudien insgesamt zu gelten scheint: Negative Ergebnisse werden häufig nicht publiziert.

Fazit: Dies ist die erste Analyse, die sich auf prospektiv erhobene Studiendaten stützt. Im Gegensatz zu früheren Untersuchungen legt sie nahe, dass der NLR weder ein prognostischer noch ein prädiktiver Biomarker für das OS bei muskelinvasivem $\mathrm{BC}$ ist. Brigitte Schalhorn

Ojerholm E et al. Neutrophil-to-lymphocyte ratio as a bladder cancer biomarker: assessing prognostic and predictive value in SWOG 8710. Cancer. 2017;123(5):794-801. 